\title{
A MAP OF THE FOURTH CHROMOSOME OF DROSOPHILA MELANOGASTER, BASED ON CROSSING OVER IN TRIPLOID FEMALES
}

\section{A. H. STURTEvant}

\section{Kerckhoff Laboratories of Biology, California Institute of Technology, Pasadena, California}

Communicated May 8, 1951

Under ordinary conditions there is so little crossing over in the fourth chromosome of Drosophila melanogaster that the usual method of constructing a map is not practicable. Deduction from the behavior of translocations has been utilized, but as will be shown here, has led to an incorrect result. Bridges and Brehme (1944) give the seriation bt (bent), sv (shaven), $c i$ (cubitus interruptus), gvl (grooveless), ey (eyeless), with 0.2 per cent crossing over for the whole series. This crossover value is certainly too high; it may be doubted if as many as five crossovers have ever been detected from diploid females. The results presented below show also that the above sequence is altogether incorrect, the true order being $c i$, $g v l, b t, e y, s v$, (with a possibility that the positions of $c i$ and $g v l$ should be reversed).

TABLE 1

\section{Crossing Over in Diplo-IV Triploid Females}

\begin{tabular}{|c|c|c|c|c|c|c|c|c|}
\hline MOTHER & 0 & 1 & 2 & 3 & 1,2 & 1,3 & 2,3 & TOTAL \\
\hline$+/ c i$ gvl ey sv & 2017 & 3 & 34 & 20 & 1 & 1 & 1 & 2077 \\
\hline gvl sv/ci ey & 305 & $\underline{0}$ & 4 & 2 & $\underline{0}$ & $\underline{0}$ & $\underline{0}$ & 311 \\
\hline Total & $\overline{2322}$ & $\overline{3}$ & $\overline{38}$ & $\overline{22}$ & $\overline{1}$ & $\overline{1}$ & $\overline{1}$ & 2388 \\
\hline \multicolumn{9}{|c|}{ Tested Crossovers } \\
\hline$+/ c i$ gvl ey sv & & 2 & 23 & 12 & 0 & 0 & 0 & \\
\hline gol sv/ci ey & & 0 & 3 & 2 & 0 & 0 & 0 & \\
\hline Total & & 2 & 26 & 14 & 0 & 0 & 0 & \\
\hline
\end{tabular}

I have reported (Morgan, Sturtevant and Morgan, 1945) that there is an appreciable amount of crossing over in the fourth chromosomes of diplo-IV triploid females. This has made it possible to build up multiple recessive stocks, which have now been used in four-point test-cross experiments (table 1). (The mutant alleles used were $c i, g v l, e y^{\mathrm{R}}$, and $s v^{\mathrm{na}}$.) The crossover classes are designated by regional numbers based on the assumption that the sequence is as given, and are clearly inconsistent with any other sequence, except that for region 1 (between $c i$ and $g v l$ ) there are three single crossovers and two doubles, which means that the seriation $g v l, c i, e y, s v$ would give nearly as good a fit.

It happens that $c i=$ and to a lesser extent gvl and ey-may at times be 
somewhat difficult to classify with certainty. I have, therefore, carried out genetic tests on many of these crossovers (usually by mating them to ci gvl ey sv, but in some cases by getting individuals homozygous for the crossover chromosome). In these tests the determination was, in each case, based on numerous individuals, thereby eliminating the uncertainty of classification. As the table shows, no such test was obtained on any of the three individuals recorded as double crossovers; it may be that all of them were wrongly classified-though the evidence now to be presented makes this unlikely.

Before the $c i$ gvl ey sv stock was available several crosses were carried out in which females heterozygous for all four loci were test-crossed to males with two of the four recessives, and the crossovers between these two were then tested both for confirmation of the classification with respect to these two, and also for the presence of the other two recessives. These data do not permit any simple direct calculation of crossover values, but as Table 2 shows, they do include four tested double crossovers. The

TABLE 2

\begin{tabular}{|c|c|c|c|c|c|c|c|}
\hline \multicolumn{8}{|c|}{ Tested Crossovers from 4-Point Test-Crosses } \\
\hline MOTHER & FATHER & 1 & 2 & 3 & 1,2 & 1,3 & 2,3 \\
\hline gvl sv/ci ey & ciey & 0 & 2 & 0 & 0 & 0 & 0 \\
\hline gvl sv/ci ey & gvl ey & 0 & 6 & 0 & 0 & 0 & 1 \\
\hline gvl sv/ci ey & gvl sv & 0 & 9 & 1 & 0 & 0 & 0 \\
\hline$g v l$ ey/ci sv & $c i s v$ & 0 & 2 & 1 & 0 & 0 & 0 \\
\hline gvl ey/ci sv & gul ey & $\underline{0}$ & 6 & $\mathbf{0}$ & 1 & $\mathbf{0}$ & 2 \\
\hline Total & & $\overline{0}$ & $\overline{25}$ & $\overline{2}$ & $\overline{1}$ & $\overline{0}$ & 3 \\
\hline Table 1 & & 2 & 26 & 14 & 0 & 0 & 0 \\
\hline Grand tota & & $\overline{2}$ & $\overline{51}$ & $\overline{16}$ & $\overline{1}$ & $\overline{0}$ & $\overline{3}$ \\
\hline
\end{tabular}

occurrence of true doubles thus seems clear, though it is of course wholly unexpected when the total amount of crossing over is so low. No satisfactory explanation of this high coincidence has yet been produced.

Table 2 shows one 1.2 double, which suggests that the true sequence is gvl $c i$ ey $s v$. It is to be noted, however, that none of the experiments of table 2 involved the use of $c i$ gvl males for test-crossing, with the result that single crossovers in region 1 (if the sequence is ci gvl ey sv) could have been detected only in the tests to $c i$ males. These included only five of the 31 tested crossovers.

In some of the cultures included in the first row of table 1 , the "+" chromosome actually carried the gene $b t$. In these cases most of the crossovers were tested not only. for $c i, g v l$, ey and $s v$, but also for $b t$, with the results shown in table 3 .

These tests (which are all included in the lower half of table 1, disregarding $b t$ ) show that $b t$ lies between $g v l$ and $e y$, and is probably nearer ey. This conclusion is not in agreement with the locus usually given 
(Dubinin, Sokolov and Tiniakov 1935, Bridges and Brehme 1944). The incorrect locus seems to have arisen from acceptance of the report of Bolen (1931) that the break in translocation white-mottled-5 lies between the loci of $e y$ and of $b t$. I have recently reinvestigated this translocation, which involves breaks near the base of IV and the tip of X. There is a viable hyperploid type, which carries a duplication for the tip of $\mathrm{X}$ and the base of IV. Bolen reported that this duplication did not suppress the eyeless phenotype when added to flies of the composition ey/ey, but did suppress bent in flies of the composition $b t / b t$. I can confirm the result for $e y$, and can extend it to $g v l, s v$ - and $b t$. The duplication males have spread wings, which makes classification for $b t$ rather uncertain in them; but I have seen a duplication-carrying female with all the various aspects of the best phenotype fully expressed.

Dubinin, Sokolov and Tiniakov (1935) concluded that the "heterochromatic effect" or a translocation could be spread through the $b t$ locus without affecting the bent phenotype; the present results, showing that $b t$ was wrongly located, indicate that their data have no bearing on this

TABLE 3

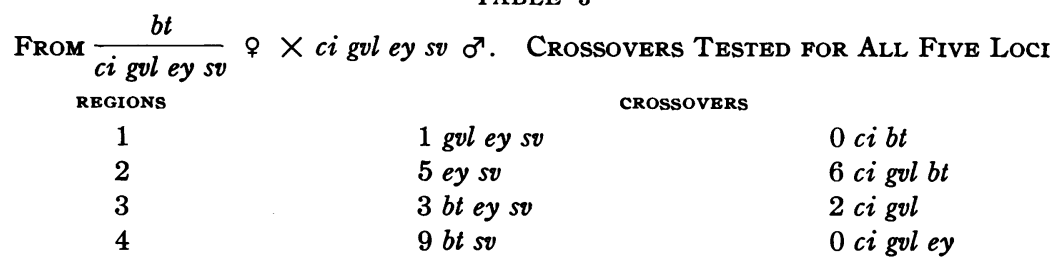

question. It should be added that the statement by Bridges and Brehme (1944) that white-mottled-5 "when tested over $c i$, shows no position effect on dominance of $c i^{+}$" is incorrect, as had already been reported by Dubinin, Sokolov and Tiniakov (1935)-a result confirmed in the present work. The position effect is present in rather extreme form.

Summary. - A map of the fourth chromosome of Drosophila melanogaster, based on crossing over in diplo-IV triploid females, shows the following relations (calculated from the upper half of table 1 , with $b t$ inserted on the basis of the data of table 3$)$ : $c i(0) ; g v l(0.2) ; b t(1.4)$; ey (2.0); sv (3.0).

The sequence shown is definitely established except that it is still possible (though unlikely) that $c i$ and gvl should be reversed. The uncertainty arises from the occurrence of unexpected double crossover classes.

The sequence given is in agreement with those reached by Fung and Stern in the accompanying paper.

Bolen, H. R., Am. Nat., 65, 417-422 (1931).

Bridges, C. B., and Brehme, K. S., Carnegie Inst. Wash. Pub. 552, 1944, 253 pp.

Dubinin, N. P., Sokolov, N. N., and Tiniakov, G. G., Biol. Zhurn., 4, 716-720 (1935).

Morgan, T. H., Sturtevant, A. H., and Morgan, L. V., Carnegie Inst. Wash. Yearbook, 44, 157-160 (1945). 